\title{
Analysis of Non-Isothermal MHD Thin Film Flow from Moving Belt with Slip and Variable Viscosity
}

\author{
A. Shahzad ${ }^{1}$, Shamsul Haq ${ }^{2 *}$ and Muhammad Ilyas ${ }^{3}$ \\ 1,2,3 Department of Mathematics, COMSATS University Islamabad, Abbottabad Campus, Pakistan \\ * Corresponding author
}

\begin{abstract}
This paper aims the study of electrically conducting Newtonian fluid flow and heat transfer considering the slip at the moving belt with temperature dependent viscosity. Adomian decomposition method (ADM) is employed to solve the non-linear system of equations. Explicit expression are obtained for velocity profile and temperature distribution. Effect of variable viscosity parameter, slip, Hartmann number, Brinkmann number and Stoke number are discussed and graphically shown.
\end{abstract}

Keywords: Slip condition; $M H D$; ADM; variable viscosity

2010 Mathematics Subject Classification: 76Axx; 76Dxx; 76Bxx; 93A30; 80Axx.

\section{Introduction}

Hydromagnetic fluid flow under the influence of viscous forces and heat transfer are of great importance in understanding a variety of geophysical, plasma aerodynamics, mechanical engineering manufacturing processes and MHD energy system [1,2]. The flow of incompressible viscous fluid between parallel plates channels in the presence of transverse magnetic field was first studied by Hartmann [3]. Attia et al [4] numerically investigated the effect of an external uniform magnetic field as well as the action of an inflow perpendicular to the plates together with the influence of the pressure gradient on the flow and temperature distributions assuming temperature dependent viscosity. In all the study except [4], the physical properties are considered to be constant. However, this may not happen in several application where the physical properties such as viscosity, thermal conductivity, density etc, may change with temperature. The effect of temperature dependent viscosity and thermal conductivity on the classical couette flow between parallel plates is investigated by Panktokratoras [5]. The same effect are also studied in micropolar fluid past a continuously moving plate with suction and injection in the presence of magnetic field is studied by Patowary et al [6]. Makinde [7] investigated thin film flow along a heated inclined plate due to the hydrodynamically and thermally developed temperature dependent viscosity of Newtonian fluid. In all the mentioned studies, the effect of slip condition is not considered.

Most of the MHD flow with the assumption of temperature dependent viscosity yields the nonlinear system of equations. The exact solution of these system are limited, and these nonlinear equations should be solved either numerically or semi- exact analytical methods. In this paper, we introduced Adomian decomposition method (ADM) [9, 10] to solve the nonlinear system with slip presented by Navier [8] at the boundary. To the best of our knowledge, no attention has been given to study the effect of slip and temperature dependent viscosity MHD on Newtonian fluid flow from moving heated belt. Thus, the primary objective of this work is to present the analytical solution of the mentioned problem by ADM $[9,10]$. 


\section{Governing Equations}

The basic equations governing the motion of steady, incompressible and non-isothermal MHD Newtonian fluid are

$$
\begin{aligned}
\nabla . \mathbf{V} & =0 \\
\rho \frac{D \mathbf{V}}{D t} & =-\nabla p+\nabla \tau+\rho \mathbf{f}-\sigma B_{0}^{2} \mathbf{V}, \\
\rho C_{p} \frac{D T}{D t} & =k \nabla^{2} T+\tau . \mathbf{L},
\end{aligned}
$$

where $\mathbf{V}$ is the velocity vector, $\rho$ is the density, $p$ is the pressure, $\mathbf{f}$ is the body force per unit mass, $B_{0}$ is the components of magnetic field, $\sigma$ is the electrical conductivity, $C_{p}$ is specific heat, $T$ is the temperature, $k$ is thermal conductivity, $\tau$ is extra stress tensor and $D / D t$ is the material time derivative. The constitutive equation for Newtonian fluid is

$$
\tau=\mu \mathbf{A}_{1}, \quad \mathbf{A}_{\mathbf{1}}=(\nabla \mathbf{V})+(\nabla \mathbf{V})^{T},
$$

where $\mu$ is the viscosity, $\mathbf{A}_{1}$ is the first Rivlin-Ericksen tensor and the superscript ${ }^{T}$ stands for the transpose of tensor.

\section{Formulation of Problem}

Consider a container filled with non-isothermal and incompressible electrically conducting (MHD) Newtonian fluid. Slippery and heated belt is assumed that is moving vertically upward through this container with constant speed $U$ (Fig.1). The belt is maintained at a constant temperature $T_{w}$ and $T_{a}$ is the free stream temperature with $T_{w}>T_{a}$. Since the belt moves upward and passes through the fluid, it picks up a thin film fluid of thickness $\delta$. Due to gravity, the fluid film tends to drain down the belt.

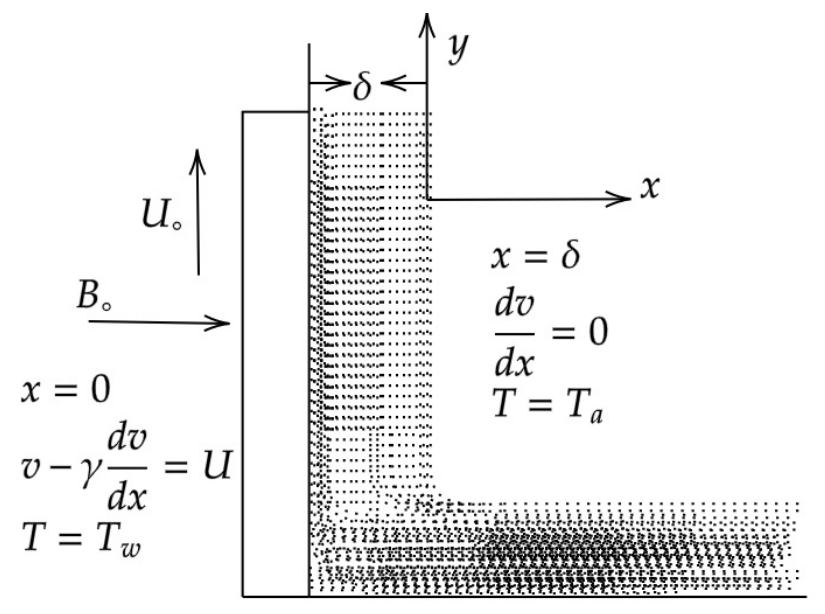

Figure 3.1: Geometry of the problem

For simplicity we assume that:

1. The flow steady, laminar and uniform.

2. The film thickness $\delta$ is uniform.

4. The applied magnetic field $B_{0}$ is transverse to the belt.

5. The thermal conductivity $k$ is constant.

6 . The pressure is considered to be atmospheric $p_{0}$ at the surface.

7. The viscosity $\mu$ of the fluid is considered to be a function of temperature.

We choose rectangular coordinates system having the $y$-axis along the belt in the upward direction while $x$-axis normal to it. The applied magnetic field $B_{0}$ is in $x$ direction. We seek the velocity and temperature profiles of the following form

$$
\mathbf{V}=(0, v(x), 0), \quad T=T(x), \quad \text { employing } \quad \tau=\tau(x) .
$$


Substituting (3.1) in (2.1), equation of continuity is identically satisfied and the momentum reduce to the following form

$$
\begin{aligned}
\frac{d \tau_{x x}}{d x}+\rho f_{x} & =\frac{\partial p}{\partial x} \\
\frac{d \tau_{x y}}{d x}+\rho f_{y}-\sigma B_{0}^{2} v & =\frac{\partial p}{\partial y}
\end{aligned}
$$

where $f_{x}$ and $f_{y}$ are body forces in $x$ and $y$ directions. Since the $y$-coordinate is in upward direction and gravitational force is in downward direction, so $f_{x}=0$ and $f_{y}=-g$, and above equations (3.2 -3.3) takes the form

$$
\begin{aligned}
\frac{d \tau_{x x}}{d x} & =\frac{\partial p}{\partial x} \\
\frac{d \tau_{x y}}{d x}-\rho g-\sigma B_{0}^{2} v & =\frac{\partial p}{\partial y} .
\end{aligned}
$$

Similarly, energy equation (2.3) becomes

$$
k \frac{d^{2} T}{d x^{2}}+\tau_{x y} \frac{d v}{d x}=0
$$

Using profile (3.1) in (2.4), the components of extra stress tensor $\tau$ are obtained as :

$$
\tau_{x y}=\tau_{y x}=\mu\left(\frac{d v}{d x}\right), \quad \tau_{x x}=\tau_{y y}=0 .
$$

The boundary conditions are

$$
\begin{array}{r}
v-\gamma \frac{d v}{d x}=U, \quad T=T_{w}, \quad \text { at } \quad x=0, \\
\frac{d v}{d x}=0, \quad T=T_{a}, \quad \text { at } \quad x=\delta,
\end{array}
$$

where $\gamma$ represents the slip coefficient. By substituting $\tau_{x x}=0$ in (3.4), we find that $P=P(y)$ only and we assume that the pressure $P$ is atmospheric pressure $p_{0}$, so $\frac{\partial p}{\partial y}=0$, Therefore by substituting $\tau_{x y}$ from equation (3.7), equation (3.5) and (3.6) becomes

$$
\begin{aligned}
\frac{d}{d x}\left[\mu\left(\frac{d v}{d x}\right)\right]-\rho g-\sigma B_{0}^{2} v & =0, \\
k \frac{d^{2} T}{d x^{2}}+\mu\left(\frac{d v}{d x}\right)^{2} & =0,
\end{aligned}
$$

Assuming that the viscosity of the fluid is temperature dependent, such that

$$
\mu=\mu(T)=\mu_{0} e^{-\alpha\left(T-T_{a}\right)} \approx \mu_{0}\left\{1-\alpha\left(T-T_{a}\right)\right\}, \quad \text { for } \quad \alpha<<1,
$$

where $\mu_{0}$ is the fluid viscosity at ambient temperature $T_{a}$ and $\alpha$ is the coefficient of variable viscosity that determine the strength between viscosity $\mu$ and temperature $T$. Substituting equation (3.12) in (3.10) and (3.11), we get

$$
\begin{aligned}
\frac{d}{d x}\left[\mu_{0}\left\{1-\alpha\left(T-T_{a}\right)\right\}\left(\frac{d v}{d x}\right)\right]-\rho g-\sigma B_{0}^{2} v & =0 \\
k \frac{d^{2} T}{d x}+\mu_{0}\left\{1-\alpha\left(T-T_{a}\right)\right\}\left(\frac{d v}{d x}\right)^{2} & =0 .
\end{aligned}
$$

Introducing the following dimensionless parameters

$$
\begin{aligned}
v^{*} & =\frac{v}{U}, \quad x^{*}=\frac{x}{\delta}, \quad \theta=\frac{T-T_{a}}{T_{w}-T_{a}}, \quad \beta=\alpha\left(T_{w}-T_{a}\right), \\
S_{t} & =\frac{\rho g \delta^{2}}{U \mu_{0}}, \quad M=\frac{\sigma B_{0}^{2} \delta^{2}}{\mu_{0}}, \quad B_{r}=\frac{\mu_{0} U^{2}}{k \delta^{2}\left(T_{w}-T_{a}\right)}, \quad \gamma^{*}=\frac{\gamma}{\delta} .
\end{aligned}
$$


where $\beta, S_{t}, M, B_{r}$ and $\gamma^{*}$ are variable viscosity parameter, Stokes number, magnetic parameter, Brinkman number and slip parameter. After removing $*$, we obtain the following dimensionless nonlinear differential equations

$$
\begin{array}{r}
\frac{d^{2} v}{d x^{2}}-\beta \theta \frac{d^{2} v}{d x^{2}}-\beta\left(\frac{d v}{d x}\right)\left(\frac{d \theta}{d x}\right)-S_{t}-M v=0 \\
\frac{d^{2} \theta}{d x^{2}}+B_{r}\left(\frac{d v}{d x}\right)^{2}-B_{r} \beta \theta\left(\frac{d v}{d x}\right)^{2}=0
\end{array}
$$

with boundary conditions

$$
\begin{aligned}
v-\gamma \frac{d v}{d x} & =1, \quad \theta=1, \quad \text { at } \quad x=0, \\
\frac{d v}{d x} & =0, \quad \theta=0, \quad \text { at } \quad x=1 .
\end{aligned}
$$

The exact solution of above non-linear system of equation along with the boundary conditions seems impossible. So, in order to solve the above system, Adomian decomposition method (ADM) will be used.

\section{Solution by ADM}

According to ADM, the differential equations (3.16)-(3.17) can be written in operator form as

$$
\begin{aligned}
& L v=S_{t}+M v+\beta \theta \frac{d^{2} v}{d x^{2}}+\beta\left(\frac{d v}{d x}\right)\left(\frac{d \theta}{d x}\right), \\
& L \theta=B_{r} \beta \theta\left(\frac{d v}{d x}\right)^{2}-B_{r}\left(\frac{d v}{d x}\right)^{2},
\end{aligned}
$$

where $L=\frac{d^{2}}{d x^{2}}$ is the differential operator and therefore applying the inverse operator $L^{-1}=\iint(*) d x d x$ on both sides of equation (4.1) and (4.2), we get

$$
\begin{aligned}
& v(x)=a x+b+S_{t} \frac{x^{2}}{2}+M L^{-1}(v)++\beta L^{-1}\left[\theta \frac{d^{2} v}{d x^{2}}\right]+\beta L^{-1}\left[\left(\frac{d v}{d x}\right)\left(\frac{d \theta}{d x}\right)\right], \\
& \theta(x)=c x+d+B_{r} \beta L^{-1}\left[\theta\left(\frac{d v}{d x}\right)^{2}\right]-B_{r} L^{-1}\left[\left(\frac{d v}{d x}\right)^{2}\right]
\end{aligned}
$$

where $a, b, c$ and $d$ are constant of integration to be determined. Adomian decomposition method defines the solution $u$ and $\theta$ by an infinite series of components, in the following form

$$
\begin{aligned}
& v(x)=\sum_{n=0}^{\infty} v_{n}(x), \\
& \theta(x)=\sum_{n=0}^{\infty} \theta_{n}(x),
\end{aligned}
$$

The non-linear terms $\theta \frac{d^{2} v}{d x^{2}},\left(\frac{d v}{d x}\right)\left(\frac{d \theta}{d x}\right), \theta\left(\frac{d v}{d x}\right)^{2}$ and $\left(\frac{d v}{d x}\right)^{2}$ are decomposed by a series of Adomian polynomials

$$
\begin{aligned}
\theta \frac{d^{2} v}{d x^{2}}=\sum_{n=0}^{\infty} A_{n}, & \left(\frac{d v}{d x}\right)\left(\frac{d \theta}{d x}\right)=\sum_{n=0}^{\infty} B_{n}, \\
\theta\left(\frac{d v}{d x}\right)^{2}=\sum_{n=0}^{\infty} C_{n}, & \left(\frac{d v}{d x}\right)^{2}=\sum_{n=0}^{\infty} D_{n} .
\end{aligned}
$$


Substituting (4.6), (4.7) and (4.8) in (4.3) and (4.4), we have

$$
\begin{aligned}
\sum_{n=0}^{\infty} v_{n}(x) & =a x+b+S_{t} \frac{x^{2}}{2}+M L^{-1}\left(\sum_{n=0}^{\infty} v_{n}(x)\right)+\beta L^{-1}\left(\sum_{n=0}^{\infty} A_{n}\right) \\
& +\beta L^{-1}\left(\sum_{n=0}^{\infty} B_{n}\right), \\
\sum_{n=0}^{\infty} \theta_{n}(x) & =c x+d+B_{r} \beta L^{-1}\left(\sum_{n=0}^{\infty} C_{n}\right)-B_{r} L^{-1}\left(\sum_{n=0}^{\infty} D_{n}\right) .
\end{aligned}
$$

Here are first few Adomian polynomials $A_{n}, B_{n}, C_{n}$ and $D_{n}$

$$
\begin{aligned}
A_{0}=\theta_{0} \frac{d^{2} v_{0}}{d x^{2}}, & A_{1}=\theta_{0} \frac{d^{2} v_{1}}{d x^{2}}+\theta_{1} \frac{d^{2} v_{0}}{d x^{2}} \\
B_{0}= & \left(\frac{d v_{0}}{d x}\right)\left(\frac{d \theta_{0}}{d x}\right), \\
B_{1}= & \left(\frac{d v_{0}}{d x}\right)\left(\frac{d \theta_{1}}{d x}\right)+\left(\frac{d v_{1}}{d x}\right)\left(\frac{d \theta_{0}}{d x}\right), \\
C_{0}= & \theta_{0}\left(\frac{d v_{0}}{d x}\right)^{2}, \\
C_{1}= & \theta_{1}\left(\frac{d v_{0}}{d x}\right)^{2}+2 \theta_{0}\left(\frac{d v_{0}}{d x}\right)\left(\frac{d v_{1}}{d x}\right), \\
D_{0}= & \left(\frac{d v_{0}}{d x}\right)^{2}, \\
D_{1}= & 2\left(\frac{d v_{0}}{d x}\right)\left(\frac{d v_{1}}{d x}\right) .
\end{aligned}
$$

To construct the recursive relation needed for the determination of the components $v_{0}, \theta_{0}, v_{1}, \theta_{1}, v_{2}, \theta_{2}$ and so on. we identify the zeroth components of

$$
\begin{aligned}
& v_{0}(x)=a x+b+S_{t} \frac{x^{2}}{2}, \\
& \theta_{0}(x)=d x+e
\end{aligned}
$$

and the next components are

$$
\begin{aligned}
& v_{n+1}(x)=M L^{-1} v_{n}+\beta L^{-1} A_{n}+\beta L^{-1} B_{n}, \quad n \geq 0, \\
& \theta_{n+1}(x)=B_{r} \beta L^{-1} C_{n}-B_{r} L^{-1} D_{n}, \quad n \geq 0 .
\end{aligned}
$$

Set the boundary conditions (3.18) and (3.19) into a decomposition series (4.6) and (4.7)

$$
\begin{aligned}
\sum_{n=0}^{\infty} v_{n}(x)-\gamma \frac{d}{d x} \sum_{n=0}^{\infty} v_{n}(x) & =1, \quad \sum_{n=0}^{\infty} \theta_{n}(x)=1, \quad \text { at } \quad x=0, \\
\frac{d}{d x} \sum_{n=0}^{\infty} v_{n}(x) & =0, \quad \sum_{n=0}^{\infty} \theta_{n}(x)=0, \quad \text { at } \quad x=1 .
\end{aligned}
$$

The recursive relation for the boundary conditions are obtained as

$$
\begin{aligned}
v_{0}(0)+\gamma \frac{d v_{0}(0)}{d x} & =1, \quad \theta_{0}(0)=1, \\
\frac{d v_{0}(1)}{d x} & =0, \quad \theta_{0}(1)=0,
\end{aligned}
$$


and the next components of boundary conditions are

$$
\begin{aligned}
v_{n+1}(0)+\gamma \frac{d v_{n+1}(0)}{d x} & =0, \quad \theta_{n+1}(0)=0, \quad n \geq 0, \\
\frac{d v_{n+1}(1)}{d x} & =0, \quad \theta_{n+1}(1)=0, \quad n \geq 0 .
\end{aligned}
$$

Invoking the boundary conditions (4.24) and (4.25), we get

$$
\begin{aligned}
& v_{0}(x)=1+S_{t}\left(\frac{1}{2} x^{2}-x-\gamma\right) \\
& \theta_{0}(x)=1-x .
\end{aligned}
$$

We notice that as $\gamma \rightarrow 0$, the velocity profile of viscous fluid without slip condition is obtained. The next component $v_{1}(x), \theta_{1}(x), v_{2}(x)$ and $\theta_{2}(x)$ can be determined by considering the successive iterates of the recursive relations (43)-(44) and (49)-(50) in the following form

$$
\begin{aligned}
v_{1}(x) & =M S_{t}\left[\frac{1}{4 !} x^{4}-\frac{1}{3 !} x^{3}-\frac{\gamma}{2} x^{2}+\frac{1}{3} x+\gamma x+\frac{\gamma}{3}+\gamma^{2}\right]+M\left[\frac{1}{2} x^{2}-x-\gamma\right] \\
& -\beta S_{t}\left[\frac{1}{3} x^{3}-x^{2}+x+\gamma\right] \\
\theta_{1}(x) & =-B_{r} S_{t}^{2}\left[\beta\left(\frac{1}{20} x^{5}-\frac{1}{4} x^{4}+\frac{1}{2} x^{3}-\frac{1}{2} x^{2}+\frac{1}{5} x\right)+\left(\frac{1}{12} x^{4}-\frac{1}{3} x^{3}\right.\right. \\
& \left.\left.+\frac{1}{2} x^{2}-\frac{1}{4} x\right)\right]
\end{aligned}
$$




$$
\begin{aligned}
& v_{2}(x)=S_{t}^{3} B_{r} \beta^{2}\left[-\frac{x^{7}}{140}+\frac{x^{6}}{20}-\frac{3 x^{5}}{20}+\frac{x^{4}}{4}+\frac{x^{2}}{10}-\frac{7}{30}\right]+S_{t}^{3} B_{r} \beta\left[-\frac{x^{6}}{72}+\frac{x^{5}}{12}-\frac{5 x^{4}}{24}\right. \\
& \left.+\frac{1}{4}-\frac{x^{2}}{8}\right]+S_{t} M^{2}\left[\frac{x^{6}}{720}-\frac{x^{5}}{120}-\frac{\gamma}{24} x^{4}+\left(\frac{1}{18}+\frac{\gamma}{6}\right)+\left(\frac{\gamma}{6}+\frac{\gamma^{2}}{2}\right) x^{2}\right. \\
& \left.-\left(\frac{2}{15}+\gamma^{2}+\frac{2 \gamma}{3}\right) x-\gamma^{3}-\frac{2 \gamma}{15}-\frac{2 \gamma^{2}}{3}\right]+S_{t} \beta M\left[-\frac{x^{5}}{20}+\frac{x^{4}}{4}+\left(\frac{\gamma}{3}-\frac{1}{3}\right)\right. \\
& \left.-\left(\frac{3 \gamma}{2}+\frac{1}{6}\right) x^{2}+\left(2 \gamma+\frac{7}{12}\right)+\frac{7 \gamma}{12}+2 \gamma^{2}\right]+M^{2}\left[\frac{x^{4}}{24}-\frac{x^{3}}{6}-\frac{\gamma}{2} x^{2}+\frac{x}{3}+\gamma^{2}\right. \\
& \left.+\gamma x+\frac{\gamma}{3}\right]+M \beta\left[-\frac{x^{3}}{3}+x^{2}-x-\gamma\right]+S_{t} \beta^{2}\left[-1+\frac{3 x^{2}}{2}-x-\gamma+\frac{x^{4}}{4}\right], \\
& \theta_{2}(x)=S_{t}^{4} B_{r}^{2} \beta^{2}\left[-\frac{x^{9}}{1440}+\frac{x^{8}}{160}-\frac{x^{7}}{40}+\frac{7 x^{6}}{120}-\frac{17 x^{5}}{200}+\frac{3 x^{4}}{4}-\frac{x^{3}}{30}+\frac{x}{225}\right] \\
& +S_{t}^{4} B_{r}^{2} \beta\left[-\frac{x^{8}}{672}+\frac{x^{7}}{84}-\frac{x^{6}}{24}+\frac{19 x^{5}}{240}-\frac{x^{4}}{12}+\frac{x^{3}}{24}-\frac{x}{160}\right] \\
& +S_{t}^{2} B_{r} \beta M\left[-\frac{x^{7}}{126}+\frac{x^{6}}{18}-\left(\frac{7}{60}-\frac{\gamma}{10}\right) x^{5}+\left(\frac{1}{36}-\frac{\gamma}{2}\right) x^{4}+\left(\frac{2}{9}+\gamma\right) x^{3}\right. \\
& \left.-\left(\frac{1}{3}+\gamma\right) x^{2}+\left(\frac{2 \gamma}{5}+\frac{16}{105}\right) x\right]+S_{t}^{2} B_{r} \beta^{2}\left[\frac{x^{6}}{15}-\frac{2 x^{5}}{5}+x^{4}-\frac{4 x^{3}}{3}+x^{2}\right. \\
& \left.-\frac{x}{3}\right]+S_{t}^{2} B_{r} M\left[-\frac{x^{6}}{90}+\frac{x^{5}}{15}-\left(\frac{1}{12}-\frac{\gamma}{6}\right) x^{4}-\left(\frac{1}{9}+\frac{2 \gamma}{3}\right) x^{3}+\left(\gamma+\frac{1}{3}\right) x^{2}\right. \\
& \left.-\left(\frac{7}{36}+\frac{\gamma}{2}\right) x\right]+S_{t} B_{r} \beta M\left[-\frac{x^{5}}{10}+\frac{x^{4}}{2}-x^{3}+x^{2}-\frac{2 x}{5}\right]+S_{t}^{2} B_{r} \beta\left[\frac{x^{5}}{10}\right. \\
& \left.-\frac{x^{4}}{2}+x^{3}-x^{2}+\frac{2 x}{5}\right]+S_{t}^{2} \beta\left[-\frac{x^{5}}{20}+\frac{x^{4}}{4}-\frac{x^{3}}{2}+\frac{x^{2}}{2}-\frac{x}{5}\right]+S_{t}^{2} B_{r}\left[-\frac{x^{4}}{12}\right. \\
& \left.+\frac{x^{3}}{3}-\frac{x^{2}}{2}+\frac{x}{4}\right]+S_{t} B_{r} M\left[-\frac{x^{4}}{6}+\frac{2 x^{3}}{3}-x^{2}+\frac{x}{2}\right] \text {, }
\end{aligned}
$$

Upon summing those iterates it is observed that

$$
v(x)=v_{0}(x)+v_{1}(x)+v_{2}(x)+\ldots .
$$

and

$$
\theta(x)=\theta_{0}(x)+\theta_{1}(x)+\theta_{2}(x)+\ldots .,
$$

yields the approximate solutions of $v(x)$ and $\theta(x)$.

By the help of above expressions, one can find volumetric flow rate, average velocity and heat transfer from the surface of the belt very easily by using the following formulae

$$
\begin{array}{r}
Q=\tilde{v}=\int_{0}^{1} v d x, \\
q=-\frac{d \theta}{d x}, \quad \text { at } \quad x=0,
\end{array}
$$

where $Q$ is dimensionless flow rate, $\tilde{v}$ is dimensionless average velocity and $q$ represents the rate of heat flux.

\section{Result Discussions}

In this paper, thin film flow and heat transfer from moving slippery and heated belt with temperature dependent viscosity are analyzed. Results are expressed graphically in Figs. (2-11). 
Fig (2-3) show the effect of variable viscosity parameter $\beta$ on the velocity and temperature profiles for different values of $B_{r}, S_{t}, \gamma$ and $M$. In Fig. (2) it is found that velocity decreases with the increase in $\beta$. Maximum velocity is observed at the belt and minimum at the surface. From Fig.(3), it is also noted that with the increase in $\beta$, temperature decreases near the belt surface and boundary layer called as Hartmann boundary layer is observed near the surface. Fig. (4) and Fig. (5) show the effect of slip parameter $\gamma$ for fixed values of $M=0.5, \beta=0.5, S_{t}=0.5$ and $B_{r}=15$. In Fig.(4), velocity decreases with the increase in $\gamma$. It is observed that when slip is valid there is a jump at the boundary. Velocity is maximum at the belt surface and minimum at the surface. From Fig.(5), it is again noted that with the increase in $\gamma$, temperature decreases near the belt surface and boundary layer called as Hartmann boundary layer is observed near the surface. The effect of Hartmann number $M$ on velocity and temperature are depicted in Figs. (6-7). It is found that the velocity decreases with the increase in the $M$ and temperature increases with the increase in the $M$. The velocity is maximum at the belt surface and minimum at the surface. Figs. (8-9) show the effect of $B_{r}$ on velocity and temperature. Negligible effect of $B_{r}$ is observed on velocity while temperature increases with in increase of $B_{r}$. Figs. (10-11) show the effect of $S_{t}$ on velocity and temperature. The velocity decreases while temperature increases with the increase in $S_{t}$.

\section{Summary and Conclusion}

The nonlinear system of equation arising in the flow of MHD Newtonian fluid with moving slippery and heated belt with temperature dependent viscosity is solved by ADM. The effect of the physical parameters like variable viscosity parameter, slip parameter, Hartmann number, Brinkmann number and Stokes number for velocity profile and temperature distributation are discussed and graphically shown. From this study we find the following observations:

- Velocity decreases with an increase in $M, S_{t}$ and $\gamma$.

- Maximum velocity is at the surface of belt.

- Temperature increases with an increase in $M, B_{r}$ and $S_{t}$.

- Hartmann boundary layer occurs near the surface for $\beta$ and $\gamma$.

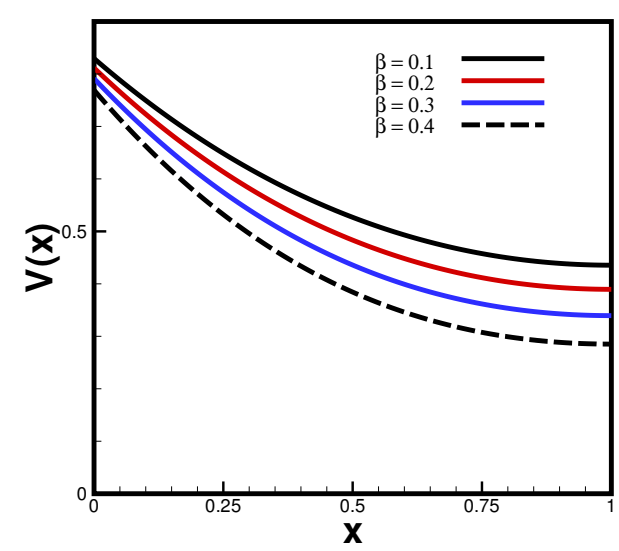

Figure 6.1: Effects of $\beta$ on flow when $M=0.5, \gamma=0.2, S_{t}=0.5$ and $B_{r}=15$

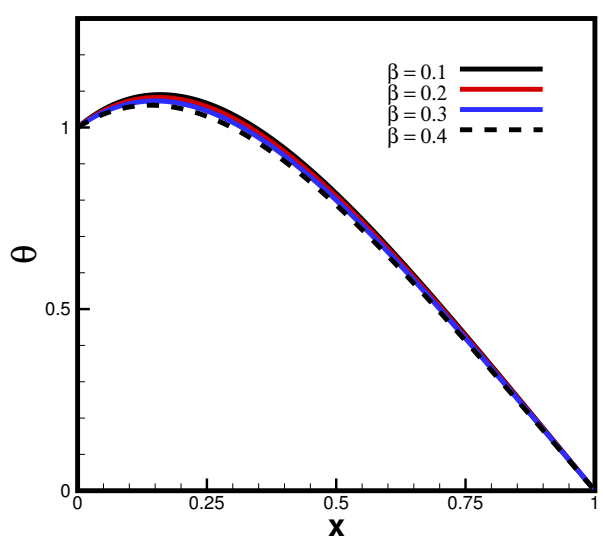

Figure 6.2: Effects of $\beta$ on temperature when $M=0.5, \gamma=0.2$, $S_{t}=0.5$ and $B_{r}=15$. 


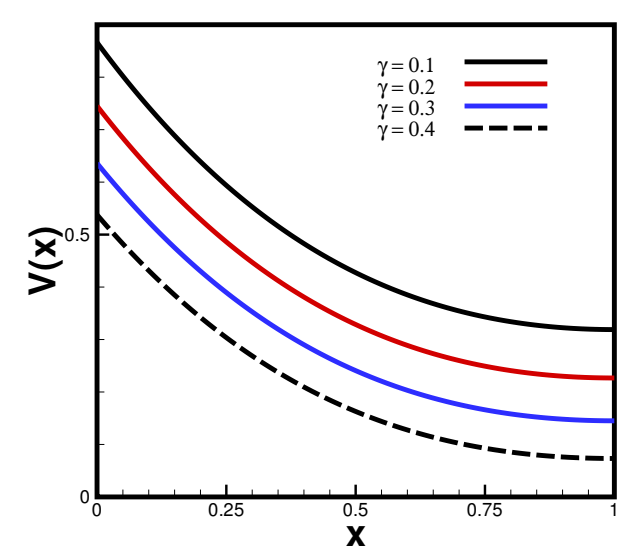

Figure 6.3: Effects of $\gamma$ on flow when $M=0.5, \beta=0.5, S_{t}=0.5$ and $B_{r}=15$

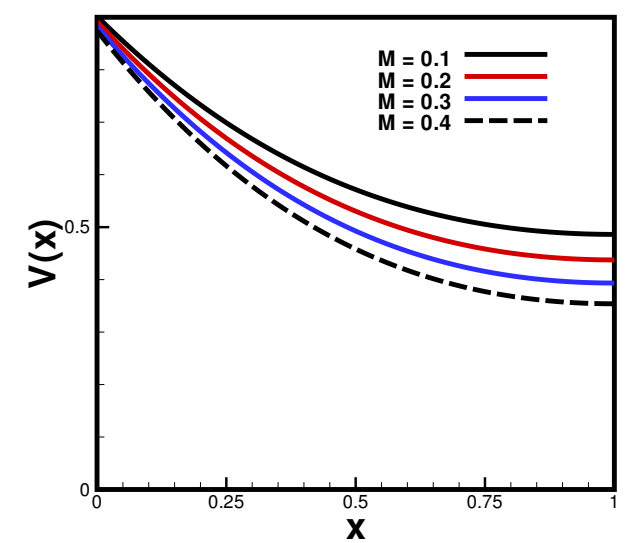

Figure 6.5: Effects of $M$ on flow when $\gamma=0.1, \beta=0.5, S_{t}=0.5$ and $B_{r}=15$

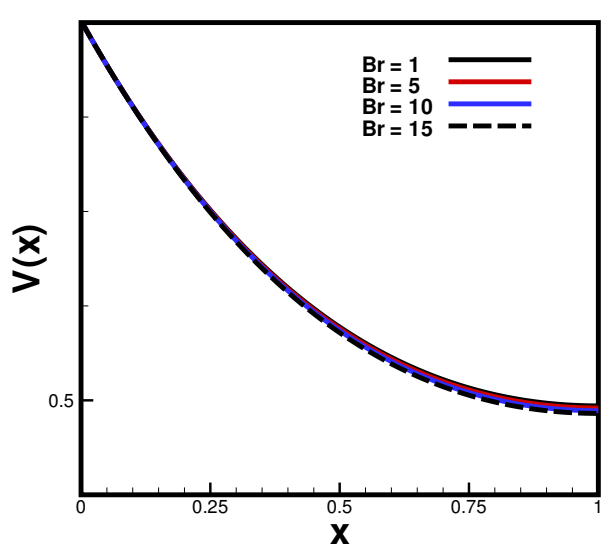

Figure 6.7: Effects of $B_{r}$ on flow when $\gamma=0.1, \beta=0.5, S_{t}=0.5$ and $M=0.1$

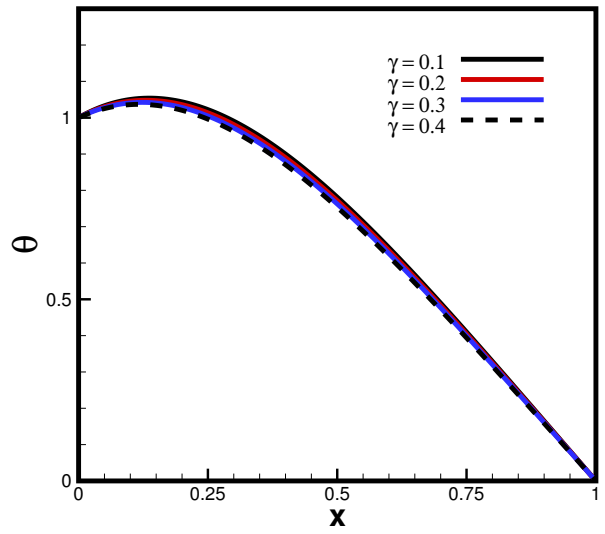

Figure 6.4: Effects of $\gamma$ on temperature when $M=0.5, \beta=0.5$, $S_{t}=0.5$ and $B_{r}=15$

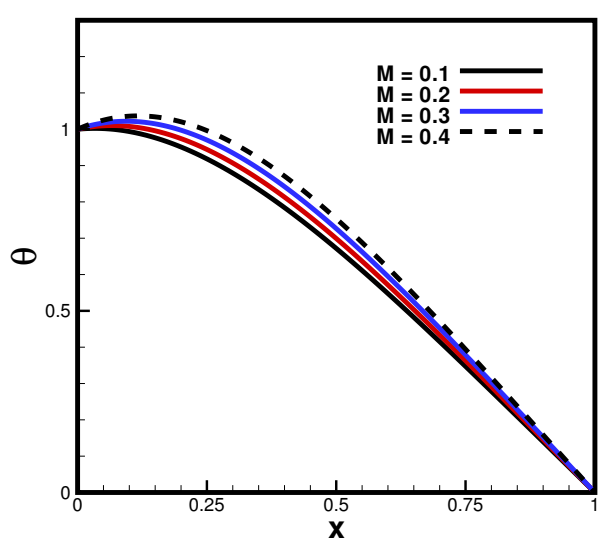

Figure 6.6: Effects of $M$ on temperature when $\gamma=0.1, \beta=0.5$, $S_{t}=0.5$ and $B_{r}=15$

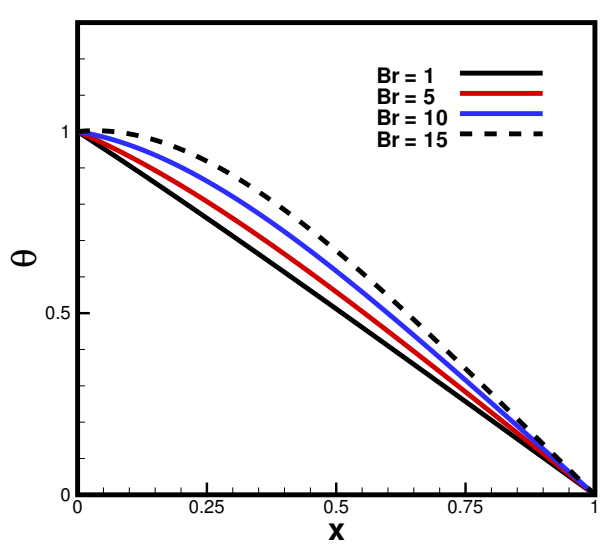

Figure 6.8: Effects of $B_{r}$ on temperature when $\gamma=0.1, \beta=0.5$, $S_{t}=0.5$ and $M=0.1$ 


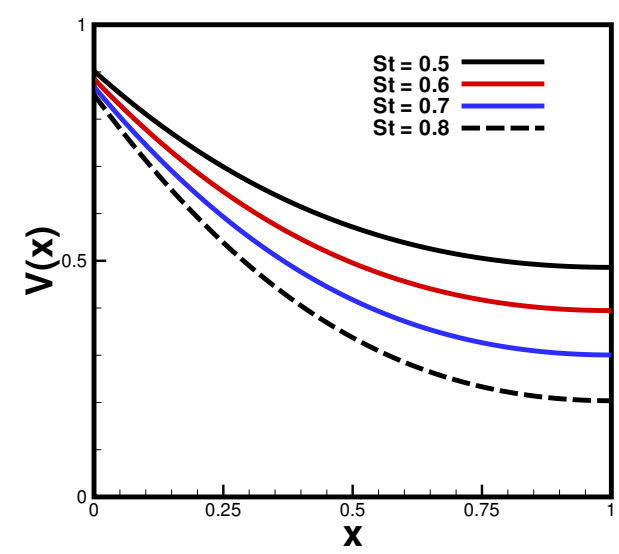

Figure 6.9: Effects of $S_{t}$ on flow when $\gamma=0.1, \beta=0.5, B_{r}=15$ and $M=0.1$

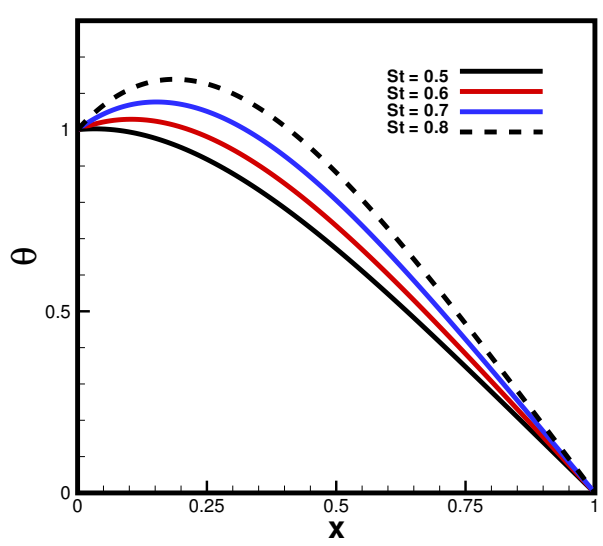

Figure 6.10: Effects of $S_{t}$ on temperature when $\gamma=0.1, \beta=0.5$, $B_{r}=15$ and $M=0.1$

\section{Acknowledgement}

Authors acknowledge Mr. M. Zubair (CUI, Abbottabad Campus) for his technical assistance.

\section{Competing Interests}

There is no actual or potential conflict of interest including any financial, personal, or other relationships with other people or organizations.

\section{References}

[1] J.A. Shercliff. A Textbook of Magnetohydrodynamics, Pergoman, London, 1965

[2] G.W. Sutton, A. Sherman. Engineering Magnetohydrodynamics, McGraw Hill Book Company, New York, 1965.

[3] J.Hartmann. Theory of laminar flow of an electrically conducting liquid in a homogeneous magnetic field, Hg-Dynamics I, Math. Fys. Med. 15, 1-28 (1937)

[4] H.A. Attia, N.A. Kotb. MHD flow between two parallel plates with heat transfer, Acta Mechanica. 117, $215-220$ (1996)

[5] A. Pantokratoras. Classical plane Couette flow with viscous dissipation and variable fluid properties, J. Thermophysics. Heat Trans. 21, 437-442 (2007)

[6] G.Patawary, D.K.Sut. Study of variable viscosity and thermal conductivity of micropolar fluid past acontinuous moving plate with suction and injection in the presence of magnetic field. Int, J.Math. Trend. Tech, 232-36 (2011).

[7] O.D. Makinde. Laminar falling liquid film with variable viscosity along an inclined heated plate. Applied Mathematics and Computation. 175, 80-88 (2006).

[8] M. Navier. Memoire sur les lois du mouvement des fluids, Mem. L'Acad.Sci. L'Inst. France. 6, 389-440 (1823).

[9] Adomian, G. A New Approach to Nonlinear Partial Differential Equations. J. Math. Anal. Appl. 102, 420-434 (1984).

[10] Adomian, G. A Review of the Decomposition Method in Applied Mathematics. J. Math. Anal. Appl. 135, 501-544 (1988). 